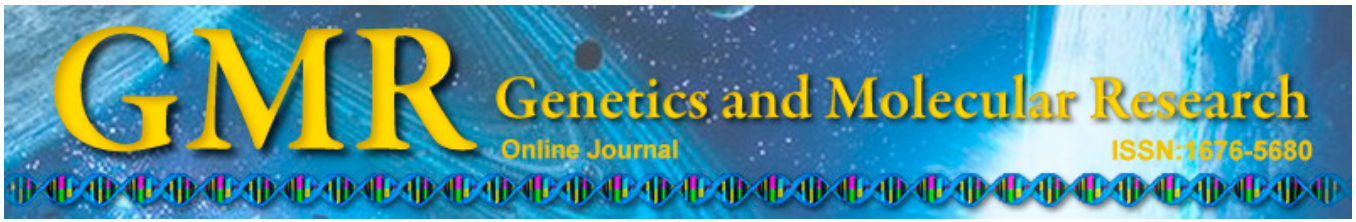

\title{
Repair of large segmental bone defects in rabbits using BMP and FGF composite xenogeneic bone
}

\author{
X. Li ${ }^{1 *}$, Z. Lin ${ }^{2 *}$, Y. Duan², X. Shu' ${ }^{2}$ A. Jin ${ }^{2}$, S. Min' and W. Yi ${ }^{1}$ \\ ${ }^{1}$ Department of Spinal Surgery, \\ Shenzhen Nanshan Hospital of Guangdong Medical College, \\ Shenzhen, Guangdong Province, China \\ ${ }^{2}$ Department of Orthopedics, Zhujiang Hospital of Southern Medical University, \\ Guangzhou, Guangdong, China \\ *These authors contributed equally to this study. \\ Corresponding author: X. Shu \\ E-mail: xiaoqiushu@126.com
}

Genet. Mol. Res. 14 (2): 6395-6400 (2015)

Received September 29, 2014

Accepted February 10, 2015

Published June 11, 2015

DOI http://dx.doi.org/10.4238/2015.June.11.15

\begin{abstract}
The objective of this study was to determine the ability of bone morphogenetic protein (BMP) and fibroblast growth factor (FGF) to repair large segmental radial bone defects in rabbits. We treated calf cancellous bones with $3 \mathrm{mg} / \mathrm{L}$ BMP (Group A), $5 \mu \mathrm{g} / \mathrm{L}$ FGF (Group B), or $3 \mathrm{mg} / \mathrm{L}$ BMP plus $5 \mu \mathrm{g} / \mathrm{L}$ FGF (Group C). A bone damage model was established using healthy radii from rabbits. The complexes were implanted in the areas of the bone defects in the radii. After successful transplantation, the rabbits underwent radiographic imaging, and bone graft specimens were detected by histopathology methods. Biomechanical indexes were also assessed in order to observe the healing status of the bone defects. Our results indicated that the repair of bone defects was significantly better in Group C compared to the other 2 groups. Therefore, we concluded that combining BMP and
\end{abstract}


FGF significantly promoted bone defect repair and achieved effects that were superior to the use of BMP alone.

Key words: Bone defect; Bone morphogenetic protein (BMP); Fibroblast growth factor (FGF)

\section{INTRODUCTION}

Treatment to repair bone defects is very difficult, and is one of the most challenging problems in orthopedics. Theoretically, autologous bone grafts are the best method for repairing bone injuries. However, clinical problems such as trauma, limited bone, and numerous sequelae caused by autologous grafts make the application of autologous bone grafts relatively limited (El-Ghannam, 2004). In recent years, there have been many studies using xenogeneic bone as a substitute material for autologous bone grafts; however, the immunogenicity and biomechanical properties of xenogeneic bone still face many problems including poor osteogenic ability, slow vascular growth, and poor repair effects on large bone injuries (Street et al., 2002; Si et al., 2012).

In this study, we observed the ability of bone morphogenetic protein (BMP) and fibroblast growth factor (FGF) complexes to repair large segmental radial bone defects in rabbits, thereby exploring a treatment for repair of large bone defects with xenogeneic bone.

\section{MATERIAL AND METHODS}

\section{Manufacture of xenogenetic bone}

Fresh calf femur cancellous bones were selected, and the muscles, ligaments, and periosteums were removed. Next, bone sticks that were $3 \times 4 \times 15 \mathrm{~mm}$ were formed and washed clean. The bone sticks were deproteinized with hydrogen peroxide $(20 \%)$, degreased with ether, disinfected with ethylene oxide, and frozen dry for use. Bone sticks that were similar in size and quality were selected and treated with $3 \mathrm{mg} / \mathrm{L} \mathrm{BMP} \mathrm{(Group} \mathrm{A),} 5 \mu \mathrm{g} / \mathrm{L} \mathrm{FGF}$ (Group B), or $3 \mathrm{mg} / \mathrm{L}$ BMP plus $5 \mu \mathrm{g} / \mathrm{L}$ FGF (Group C) (Pezzatini et al., 2007).

\section{Experimental animals}

A total of 27 healthy New Zealand rabbits were used in this study. A bone defect model was established that involved $15-\mathrm{mm}$ bone defects in the radii of the rabbits. The rabbits were randomly divided into 3 groups and implanted with the aforementioned xenogeneic bone complexes (Groups A, B, and C). They were then sutured layer by layer. The rabbits were injected with penicillin $(800,000 \mathrm{U})$ twice per day for three consecutive days following the operation to avoid wound infection. The rabbits were randomly selected for observation and measurement of outcomes 1, 3, 6,12, and 16 weeks after xenogeneic bone implantation.

\section{Measurements of indexes}

Radiographs were taken and measurements assessed 1, 3, 6, 12, and 16 weeks after the operation to quantitatively analyze new bone formation in the bone defect model (Table 
1). Anatomical observations were carried out in selected rabbits after sacrificing them. The observed factors included the color, texture, and vascular formation of the bone graft area as well as the soft tissue coverage of the host bone and surrounding areas. Next, specimens from the bone implant area were taken and stained with hematoxylin and eosin (H\&E). Briefly, the specimens were fixed in 10\% formaldehyde, were washed, and then were paraffin-embedded using an automatic embedding machine. The embedded specimens were sliced at a $3-\mu \mathrm{m}$ thickness. Following deparaffinization, dehydration, and antigen retrieval, the sections were blocked in $5 \%$ bovine serum albumin at $37^{\circ} \mathrm{C}$ for $20 \mathrm{~min}$. Sections were then incubated with 1:100 diluted primary antibodies at $4^{\circ} \mathrm{C}$ overnight. Next, sections were washed in PBS, then the secondary antibody was added dropwise and specimens were incubated at $37^{\circ} \mathrm{C}$ in a water bath for $2 \mathrm{~h}$. Specimens were then washed in PBS and treated with the DAB solution, flushed completely, counterstained with hematoxylin, washed with water, treated with dehydration and transparency, and mounted on slides and observed under a microscope. Histological observations were made according to the Lane-Sandhu histological score standards (Table 2). Three-point bending stress was measured and biomechanical comparisons were made 6,12 , and 16 weeks after the operation.

Levels of the inflammatory factor interleukin-1 (IL-1) were detected in the rabbits using an enzyme-linked immunosorbent assay 3, 6, 12, and 16 weeks after the operation.

Table 1. Lane-Sandhu scoring standards for radiographs.

\begin{tabular}{lcl}
\hline & Score & Anatomical observations \\
\hline Bone formation & 0 & No bone formation \\
& 1 & Bone formation in $25 \%$ of bone defect \\
Bone synostosis & 2 & Bone formation in $50 \%$ of bone defect \\
& 3 & Bone formation in $75 \%$ of bone defect \\
Bone remodeling & 0 & Clear fracture line \\
& 2 & Fracture line partially visible \\
& 4 & Fracture line disappeared \\
& 0 & No bone remodeling \\
& 2 & Marrow cavity formation \\
\end{tabular}

Table 2. Lane-Sandhu histology scoring standards.

\begin{tabular}{llll}
\hline & Bone synostosis & Cancellous bone & Cortical bone \\
\hline 0 & No & No bone cell activity & No cortical bone growth \\
1 & Fibrous union & New bone early accumulation & Early manifestations of cortical bone growth \\
2 & Connection of bone and osteoid & Active bone aggregation & Cortical bone is forming \\
3 & Bone synostosis & Cancellous bone is rebuilding & Most of cortical bone is rebuilt \\
4 & Complete backbone regeneration & Complete cancellous bone rebuild & Complete cortical bone formation \\
\hline
\end{tabular}

\section{Statistical analysis}

All data are reported as means \pm standard deviation. Data were analyzed by using the SPSS statistical software, version 13.0 (SPSS Inc., Chicago, IL, USA). ANOVA tests were used to analyze the data. $\mathrm{P}<0.05$ was considered to be statistically significant. 


\section{RESULTS}

\section{Anatomical observations}

One week after transplantation, the graft had not yet combined with the host bone. Three weeks after transplantation, the boundary line between the graft and the host bone was relatively clear. Six weeks after transplantation, the boundary line between the graft and host bone was indistinct and callus formation had increased. Callus formation was greater in Group $\mathrm{C}$ compared to the other 2 groups 12 weeks after transplantation. The bone injury had almost healed in Group C 16 weeks after transplantation.

\section{Radiographic results}

One week after transplantation, the fracture lines in the implant area were clear and there was no obvious callus formation. Three weeks after transplantation, the density of the grafts was slightly higher than the normal bone tissue. The fracture line became blurry and there was evident callus formation in the broken end of the host bone 6 weeks after transplantation. The fracture line became very blurry and there was considerable callus formation in the broken end of the host bone 12 weeks after transplantation. The fracture line almost disappeared, healing bone was observed in the area of the defect, and the bone density of the graft was similar to the surrounding normal tissue 16 weeks after transplantation. There was a significant difference between Group C and Groups A and B $(\mathrm{P}<0.05)$.

\section{H\&E staining}

A significant increase in new capillaries was observed in Groups B and C starting 8 weeks after the operation; the effect was most obvious in Group $\mathrm{C}(\mathrm{P}<0.05)$. The microvessel density in the area of the bone defect was higher in Group $\mathrm{C}$ compared to the other 2 groups $(\mathrm{P}<0.05$, Table 3).

$\begin{aligned} & \text { Table 3. Microvessel density in the area of the bone defect at different times after bone implantation in the } \\
& \text { different groups. }\end{aligned}$
\begin{tabular}{lcccc}
\hline & Third week & Sixth week & Twelfth week & Sixteenth week \\
\hline Group A & $3.4 \pm 1.5$ & $3.9 \pm 2.3$ & $4.8 \pm 1.9$ & $6.4 \pm 2.7$ \\
Group B & $3.2 \pm 1.2$ & $4.1 \pm 2.0$ & $5.2 \pm 1.4$ & $6.2 \pm 2.5$ \\
Group C & $4.0 \pm 2.1$ & $5.3 \pm 1.8$ & $6.4 \pm 1.2^{*}$ & $7.9 \pm 2.2^{*}$ \\
\hline
\end{tabular}

$* \mathrm{P}<0.05$ compared to Group A.

\section{Biomechanical measurements}

As shown in Table 4, three-point bending stress was significantly enhanced in Group $\mathrm{C}$ compared to the other 2 groups $(\mathrm{P}<0.05)$.

\section{IL-1 levels}

Levels of IL-1 were lower in Group C compared to the other 2 groups $(\mathrm{P}<0.05)$, 
indicating that the inflammatory response was significantly lower in Group C compared to the other 2 groups (Table 5).

Table 4. Three-point bending stress at different times after bone implantation.
\begin{tabular}{lcccc}
\hline & Sixth week & Twelfth week & Sixteenth week \\
\hline Group A & Third week & $60.54 \pm 2.33$ & $63.65 \pm 1.96$ & $63.73 \pm 2.74$ \\
Group B & $61.54 \pm 1.64$ & $59.76 \pm 3.03$ & $58.93 \pm 3.99$ & $60.45 \pm 2.65$ \\
Group C & $98.35 \pm 1.92$ & $91.53 \pm 4.21^{*}$ & $93.42 \pm 2.22^{*}$ & $95.01 \pm 3.25^{*}$ \\
\hline
\end{tabular}

$* \mathrm{P}<0.05$ compared to Group A.

Table 5. Interleukin-1 levels after bone implantation.

\begin{tabular}{lllcc}
\hline & Third week & Sixth week & Twelfth week & Sixteenth week \\
\hline Group A & $5.63 \pm 1.75$ & $5.56 \pm 2.03$ & $4.35 \pm 1.69$ & $3.63 \pm 1.72$ \\
Group B & $5.75 \pm 1.35$ & $5.46 \pm 2.54$ & $4.65 \pm 1.76$ & $3.46 \pm 1.63$ \\
Group C & $4.21 \pm 1.35^{*}$ & $4.01 \pm 1.26^{*}$ & $3.57 \pm 2.01^{*}$ & $2.33 \pm 1.25^{*}$ \\
\hline
\end{tabular}

$* \mathrm{P}<0.05$ compared to Group A.

\section{DISCUSSION}

BMP is a member of the transforming growth factor- $\beta$ superfamily. First identified in 1965, BMP has been shown to be beneficial for osteogenesis induction. BMP is the strongest known osteoinductive factor. The biological effects of BMP are very important in biological body growth processes, the early stages of fractures, the ossification process in cartilage, and cartilage injury repair. Overexpression of BMP may occur in these cases, as it has significant roles in development, regeneration, and bone repair (Chen et al., 1997; Pezzatini et al., 2007; Budiraharjo et al., 2013; Fei et al., 2013).

Reestablishing blood supply is an important part of the bone graft repair process for large segmental bone defects. Under normal circumstances, blood vessels mainly grow gradually from one end of the host backbone to the graft. In this bone repair growth process, blood vessels grow slowly, and it is difficult to guarantee promotion of bone repair by local vascular growth. Currently, basic FGF and vascular endothelial growth factor are thought to be the most effective angiogenic cytokines. Many studies have indicated that FGF is an important mitogenic factor. FGF is also an important differentiation-inducing factor with broad biological functions in the cell differentiation process (Behr et al., 2012; Crowley et al., 2013). Some studies have shown that FGF promotes angiogenesis, wound healing, and tissue repair, which are important for tissue and nerve regeneration (Porter et al., 2009; Iwakura et al., 2013).

In this study, we found that FGF promotes healing and recovery of bone trauma after xenogeneic bone grafts when combined with BMP; angiogenesis was greater in the group that received both FGF and BMP compared to the groups that received only one of these factors. FGF and BMP complexes had a significant $b$ better therapeutic effect and induced lower levels of the inflammatory factor IL-1 compared to xenograft bones treated only with BMP. These results confirm that FGF is important for bone repair, as it promoted bone healing and reduced the inflammation reaction (Hou et al., 2009; Che et al., 2010). 
In summary, we used anatomical, radiographic, histological, and biomechanical methods to establish that xenogeneic bone materials made of a combination of BMP and FGF have a superior effect for the treatment of large segmental bone defects compared to BMP or FGF alone. This provides a new practical basis for the clinical treatment of bone injuries and for material development for bone grafts and repair.

\section{Conflicts of interest}

The authors declare no conflict of interest.

\section{ACKNOWLEDGMENTS}

Research supported by the Medical Research Funds of Guangdong Province in China (Contract \#B2013254) and the Natural Science Funds of China (Contract \#H0605/81201381).

\section{REFERENCES}

Behr B, Sorkin M, Lehnhardt M, Renda A, et al. (2012). A comparative analysis of the osteogenic effects of BMP-2, FGF2, and VEGFA in a calvarial defect model. Tissue Eng. Part A 18: 1079-1086.

Budiraharjo R, Neoh KG and Kang ET (2013). Enhancing bioactivity of chitosan film for osteogenesis and wound healing by covalent immobilization of BMP-2 or FGF-2. J. Biomater. Sci. Polym. Ed. 24: 645-662.

Che JH, Zhang ZR, Li GZ, Tan WH, et al. (2010). Application of tissue-engineered cartilage with BMP-7 gene to repair knee joint cartilage injury in rabbits. Knee Surg. Sports Traumatol. Arthrosc. 18: 496-503.

Chen D, Harris MA, Rossini G, Dunstan CR, et al. (1997). Bone morphogenetic protein 2 (BMP-2) enhances BMP3 , BMP-4, and bone cell differentiation marker gene expression during the induction of mineralized bone matrix formation in cultures of fetal rat calvarial osteoblasts. Calcif. Tissue Int. 60: 283-290.

Crowley C, Wong JM, Fisher DM and Khan WS (2013). A systematic review on preclinical and clinical studies on the use of scaffolds for bone repair in skeletal defects. Curr. Stem Cell Res. Ther. 8: 243-252.

El-Ghannam AR (2004). Advanced bioceramic composite for bone tissue engineering: design principles and structurebioactivity relationship. J. Biomed. Mater. Res. A 69: 490-501.

Fei Y, Gronowicz G and Hurley MM (2013). Fibroblast growth factor-2, bone homeostasis and fracture repair. Curr. Pharm. Des. 19: 3354-3363.

Hou H, Zhang X, Tang T, Dai K, et al. (2009). Enhancement of bone formation by genetically-engineered bone marrow stromal cells expressing BMP-2, VEGF and angiopoietin-1. Biotechnol. Lett. 31: 1183-1189.

Iwakura T, Sakata R and Reddi AH (2013). Induction of chondrogenesis and expression of superficial zone protein in synovial explants with TGF-beta1 and BMP-7. Tissue Eng. Part A. 19: 2638-2644.

Pezzatini S, Morbidelli L, Solito R, Paccagnini E, et al. (2007). Nanostructured HA crystals up-regulate FGF-2 expression and activity in microvascular endothelium promoting angiogenesis. Bone 41: 523-534.

Porter JR, Ruckh TT and Popat KC (2009). Bone tissue engineering: a review in bone biomimetics and drug delivery strategies. Biotechnol. Prog. 25: 1539-1560.

Si HP, Lu ZH, Lin YL, Li JJ, et al. (2012). Transfect bone marrow stromal cells with pcDNA3.1-VEGF to construct tissue engineered bone in defect repair. Chin. Med. J. 125: 906-911.

Street J, Bao M, deGuzman L, Bunting S, et al. (2002). Vascular endothelial growth factor stimulates bone repair by promoting angiogenesis and bone turnover. Proc. Natl. Acad. Sci. U. S. A. 99: 9656-9661. 